\title{
Concentration of mass on the Schatten classes
}

\author{
O. Guédon ${ }^{\mathrm{a}, *}$, G. Paouris ${ }^{\mathrm{b}, 1}$ \\ ${ }^{\text {a } U n i v e r s i t e ́ ~ P i e r r e ~ e t ~ M a r i e ~ C u r i e, ~ E ́ q u i p e ~ d ' A n a l y s e ~ F o n c t i o n n e l l e, ~ I n s t i t u t ~ d e ~ M a t h e ́ m a t i q u e s ~ d e ~ J u s s i e u, ~ b o i ̂ t e ~ 186, ~ 4, ~ P l a c e ~ J u s s i e u, ~}$ \\ 75252 Paris cedex 05, France \\ b Laboratoire d'Analyse et de Mathématiques Appliquées, Université de Marne-la-Vallée, 5, Bd Descartes, Champs-sur-Marne, \\ 77454 Marne-la-Vallée cedex 2, France
}

Received 20 May 2005; received in revised form 7 November 2005; accepted 6 January 2006

Available online 7 July 2006

\begin{abstract}
Let $1 \leqslant p \leqslant \infty$ and $\widetilde{B\left(S_{p}^{n}\right)}$ be the unit ball of the Schatten trace class of matrices on $\mathbb{C}^{n}$ or on $\mathbb{R}^{n}$, normalized to have Lebesgue measure equal to one. We prove that

$$
\lambda\left(\left\{T \in \widehat{B\left(S_{p}^{n}\right)}: \frac{\|T\|_{\mathrm{HS}}}{n} \geqslant c_{1} t\right\}\right) \leqslant \exp \left(-c_{2} t n^{k_{p}}\right)
$$

for every $t \geqslant 1$, where $k_{p}=\min \{2,1+p / 2\}, c_{1}, c_{2}>1$ are universal constants and $\lambda$ is the Lebesgue measure. This concentration of mass inside a ball of radius proportional to $n$ follows from an almost constant behaviour of the $L_{q}$ norms (with respect to the Lebesgue measure on $\widehat{B\left(S_{p}^{n}\right)}$ ) of the Hilbert-Schmidt operator norm of $T$. The same concentration result holds for every classical ensembles of matrices like real symmetric matrices, Hermitian matrices, symplectic matrices or antisymmetric Hermitian matrices. The result is sharp when $p=1$ and $p \geqslant 2$.
\end{abstract}

(C) 2006 Elsevier Masson SAS. All rights reserved.

\section{Résumé}

Pour tout $1 \leqslant p \leqslant \infty$, soit $\widetilde{B\left(S_{p}^{n}\right)}$ la boule unité des classes de Schatten à trace, normalisée pour avoir un volume égal à 1 . Nous prouvons que pour tout $t \geqslant 1$,

$$
\lambda\left(\left\{T \in \widehat{B\left(S_{p}^{n}\right)}: \frac{\|T\|_{\mathrm{HS}}}{n} \geqslant c_{1} t\right\}\right) \leqslant \exp \left(-c_{2} t n^{k_{p}}\right)
$$

où $k_{p}=\min \{2,1+p / 2\}, c_{1}, c_{2}>1$ sont des constantes universelles et $\lambda$ désigne la mesure de Lebesgue. Ce phénomène de concentration du volume à l'intérieur d'une boule euclidienne de rayon proportionnel à $n$ découle d'un comportement presque constant des normes $L_{q}$ (par rapport à la mesure de Lebesgue sur $\widehat{B\left(S_{p}^{n}\right)}$ ) de la norme Hilbert-Schmidt d'un opérateur $T$. Le même phénomène de concentration est valable pour tous les ensembles classiques de matrices : les matrices symétriques réelles, les matrices hermitiennes, les matrices symplectiques ou encore les matrices hermitiennes antisymétriques. De plus, le résultat est optimal lorsque $p=1$ et $p \geqslant 2$.

(c) 2006 Elsevier Masson SAS. All rights reserved.

\footnotetext{
* Corresponding author.

E-mail addresses: guedon@math.jussieu.fr (O. Guédon), grigoris_paouris@yahoo.co.uk (G. Paouris).

1 G. Paouris acknowledges the financial support provided through the European Community's Human Potential Programme under contract MRTN-CT-2004-511953 (Phenomena in High Dimensions).
} 


\section{Introduction}

Let $K$ be a symmetric convex body in $\mathbb{R}^{d}$, and let $\mathcal{E} \in \mathcal{E} l l=\left\{\mathcal{E}\right.$ an ellipsoid, $\left.|\mathcal{E}|=\omega_{d}\right\}$ where $|\mathcal{E}|$ denotes the volume of $\mathcal{E}$ and $\omega_{d}$ is the volume of the Euclidean unit ball in $\mathbb{R}^{d}$. For every $q \geqslant 1$ consider the $L_{q}$ normalized norm of the Euclidean norm associated to $\mathcal{E}$ :

$$
I_{q}(K, \mathcal{E})=\left(\frac{1}{|K|^{1+q / d}} \int_{K}\|x\|_{\mathcal{E}}^{q} \mathrm{~d} \lambda(x)\right)^{1 / q},
$$

where $\lambda$ is the Lebesgue measure on $\mathbb{R}^{d}$.

Conjecture (C). There exist a universal constant $C>0$ and a function $\phi(d)$ tending to infinity with $d$, which satisfy the following: for every centrally symmetric convex body $K$ in $\mathbb{R}^{d}$ there is an ellipsoid $\mathcal{E} \in \mathcal{E}$ ll such that, for all $q \leqslant \phi(d)$,

$$
\left(\frac{1}{|K|} \int_{K}\|x\|_{\mathcal{E}}^{q} \mathrm{~d} \lambda(x)\right)^{1 / q} \leqslant C\left(\frac{1}{|K|} \int_{K}\|x\|_{\mathcal{E}}^{2} \mathrm{~d} \lambda(x)\right)^{1 / 2} .
$$

Equivalently, this means that $I_{q}(K, \mathcal{E}) \leqslant C I_{2}(K, \mathcal{E})$.

The goal of this paper is to verify conjecture (C) for the case of Schatten trace classes of matrices and their subspaces which are of particular interest. Observe that for every $\alpha>0$ and every $q \geqslant 1, I_{q}(\alpha K, \mathcal{E})=I_{q}(K, \mathcal{E})$ and for every linear transformation $T \in \mathrm{SL}_{n}(\mathbb{R})$,

$$
I_{q}\left(T(K), B_{2}^{d}\right)=I_{q}\left(K,\left(T^{*} T\right)^{-1} B_{2}^{d}\right) .
$$

By (1), it is clear that in order to check the conjecture (C), our goal is to find a position of the symmetric convex body $K$ for which the desired estimates can be obtained with $\mathcal{E}=B_{2}^{d}$.

The isotropic constant $L_{K}$ of a symmetric convex body $K$ is defined by

$$
L_{K}=\min \left\{I_{2}(K, \mathcal{E}) / \sqrt{d} ; \mathcal{E} \text { an ellipsoid, }|\mathcal{E}|=\omega_{d}\right\} .
$$

It is well known that the hyperplane conjecture is equivalent to the fact that $L_{K}$ is uniformly bounded for all symmetric convex bodies (see [13]). We say that a symmetric convex body $K$ is in isotropic position when the minimum at Eq. (2) is attained by the Euclidean unit ball $B_{2}^{d}$. It is natural to expect that the conjecture (C) will be satisfied for a convex body in isotropic position.

By Borell's inequality [6], we know that for any symmetric convex body $K \in \mathbb{R}^{d}$,

$$
I_{q}\left(K, B_{2}^{d}\right) \leqslant C q I_{2}\left(K, B_{2}^{d}\right) .
$$

Moreover, if $K$ is a symmetric convex body of $\mathbb{R}^{d}$ in isotropic position, Alesker [1] proved that for every $q \geqslant 2$,

$$
I_{q}\left(K, B_{2}^{d}\right) \leqslant C \sqrt{q} I_{2}\left(K, B_{2}^{d}\right) .
$$

For general symmetric convex bodies, this is the best known estimate but it is very far from the conjecture. If we translate this result in terms of concentration of measure, it says that for every $t \geqslant 1$,

$$
\lambda\left(\left\{x \in \widetilde{K}:\|x\|_{2} \geqslant C t I_{2}\left(K, B_{2}^{d}\right)\right\}\right) \leqslant \exp \left(-t^{2}\right)
$$

where $\widetilde{K}$ denotes the homothetic image of $K$ of volume 1 .

It turns out that the claimed conjecture is equivalent to some type of concentration of mass inequality for convex bodies. This is part of Theorem 1.1 in [15], where other equivalent formulations of the question are studied. In the particular case of isotropic 1-unconditional convex bodies $K$ (1-unconditional means that for every point 
$x=\left(x_{1}, \ldots, x_{d}\right) \in K$ and $\left(\varepsilon_{1}, \ldots, \varepsilon_{d}\right) \in\{-1,1\}^{d}$, the point $\left(\varepsilon_{1} x_{1}, \ldots, \varepsilon_{d} x_{d}\right)$ belongs to $\left.K\right)$, Bobkov and Nazarov [5] proved a sharp concentration inequality for the Euclidean norm with respect to the normalized Lebesgue measure on $K$ :

Theorem. [5] There exists a universal constant $C>0$ such that for every isotropic 1-unconditional convex body $K$ in $\mathbb{R}^{d}$, if $\widetilde{K}$ denotes the homothetic image of $K$ of volume 1 , then for every $t \geqslant 1$,

$$
\lambda\left(\left\{x \in \widetilde{K}:\|x\|_{2} \geqslant C t I_{2}\left(K, B_{2}^{d}\right)\right\}\right) \leqslant \exp (-t \sqrt{d}) .
$$

But it is well known (see [13]) that $\sup \left\{L_{K}, K 1\right.$-unconditional convex bodies $\}$ is finite. So that in this case, there exists a constant $c>0$ such that $I_{2}\left(K, B_{2}^{d}\right) \leqslant c \sqrt{d}$ for all $d \geqslant 1$. The previous result states that the mass of an isotropic 1 -unconditional convex body is highly concentrated inside a Euclidean ball of radius $c \sqrt{d}$. This shows that, for this class of bodies, the conjecture (C) is true with $\phi(d)$ proportional to $\sqrt{d}$. (See Lemma 8.)

Our purpose is to show that for all $p \geqslant 1$, the unit balls of the Schatten trace classes of matrices $B\left(S_{p}^{n}\right)$ of every classical ensemble of matrices (real matrices, complex matrices, real symmetric matrices, complex Hermitian matrices, symplectic matrices or antisymmetric Hermitian matrices) share the same property, where the appropriate Euclidean norm is the Hilbert-Shmidt norm of an operator (which is the norm associated to the unit ball $B\left(S_{2}^{n}\right)$ ). As observed in [11], in the case of real and complex matrices, these unit balls are in isotropic position. However, we believe that this is not the case for every classical ensemble of matrices. For example, it is known that the situation may be different for real symmetric matrices as indicated in the paper [4]. We prove here:

Theorem 1. There exist universal constants $c_{1}, c_{2}>0$ such that for every $n \geqslant 1$, for every $1 \leqslant p \leqslant \infty$ and $2 \leqslant q \leqslant$ $c_{1} \min \left\{n^{2}, n^{1+p / 2}\right\}$,

$$
\left(\frac{1}{\left|B\left(S_{p}^{n}\right)\right|} \int_{B\left(S_{p}^{n}\right)}\|T\|_{\mathrm{HS}}^{q} \mathrm{~d} \lambda(T)\right)^{1 / q} \leqslant c_{2}\left(\frac{1}{\left|B\left(S_{p}^{n}\right)\right|} \int_{B\left(S_{p}^{n}\right)}\|T\|_{\mathrm{HS}}^{2} \mathrm{~d} \lambda(T)\right)^{1 / 2} .
$$

The same result holds if we replace $B\left(S_{p}^{n}\right)$ with $B\left(S_{p}^{n}\right) \cap E$ where $E$ is the subspace defining one of the classical ensembles of matrices.

More generally, there are universal constants $C, C^{\prime}>0$ such that for every $n \geqslant 1$, for every $1 \leqslant p \leqslant \infty$, for every $2 \leqslant q \leqslant c_{1} \min \left\{n^{2}, n^{1+p / 2}\right\}$ and every space $E$ of classical ensemble of matrices (real matrices, complex matrices, real symmetric matrices, complex Hermitian matrices, symplectic matrices or antisymmetric Hermitian matrices),

$$
C n \leqslant I_{q}\left(B\left(S_{p}^{n}\right) \cap E, B\left(S_{2}^{n}\right) \cap E\right) \leqslant C^{\prime} n .
$$

Remark. Theorem 1 states that the conjecture (C) is valid for $K=B\left(S_{p}^{n}\right) \cap E$ with $\phi\left(d_{n}\right) \simeq \min \left\{d_{n}, d_{n}^{1 / 2+p / 4}\right\}$ where $d_{n} \simeq n^{2}$ is the corresponding dimension of the convex body $K$ (see Section 1 ).

The next theorem is a translation of the previous result in terms of concentration of measure.

Theorem 2. There exist universal constants $c_{1}, c_{2}>0$ such that if $1 \leqslant p \leqslant \infty$ and $\widetilde{B\left(S_{p}^{n}\right)}$ is the unit ball of the Schatten trace class of matrices, normalized to have Lebesgue measure one, then

$$
\lambda\left(\left\{T \in \widetilde{B\left(S_{p}^{n}\right)}:\|T\|_{\mathrm{HS}} \geqslant c_{1} n t\right\}\right) \leqslant \exp \left(-c_{2} t n^{k_{p}}\right)
$$

for every $t \geqslant 1$, where $k_{p}=\min \{2,1+p / 2\}$.

The same result holds if we replace $\widetilde{B\left(S_{p}^{n}\right)}$ with $B \widetilde{\left(S_{p}^{n}\right) \cap E}$, the unit ball of $S_{p}^{n} \cap E$ normalized to have Lebesgue measure one, where $E$ is the subspace defining one of the classical ensembles of matrices.

The proof relies on the same method for general matrices in the real or complex case and for different classical ensembles of matrices. The computation of the integrals $I_{q}$ will use a method similar to the one used in [11] to prove the uniform boundedness of the isotropic constants of the unit balls of $S_{p}^{n}$ (for real or complex matrices). For 
all particular ensemble of matrices, classical change of variables reduces the computation of the integrals $I_{q}$ to the computation of integrals over $B_{p}^{n}$ with different type of densities of the form

$$
\prod_{1 \leqslant i<j \leqslant n}\left|x_{i}^{a}-x_{j}^{a}\right|^{b} \quad \prod_{i=1}^{n}\left|x_{i}\right|^{c},
$$

where $a, b$ are positive integers and $c$ is a nonnegative integer. A usual trick in convexity makes the computation of the integrals $I_{q}$ tractable. Surprisingly, some evaluations are possible and give a very sharp comparison between $I_{q}$ and $I_{2}$ whenever $q$ is not too large (depending on $n$ and $p$ ).

The organization of the paper is as follows. In Section 1, we indicate that the desired estimate of Theorem 1 can be reduced to an estimate of integrals over $\mathbb{R}^{n}$. In Section 2 we make the computations that complete the proof of Theorem 1. Finally in Section 3 we explain why Theorem 1 and Theorem 2 are equivalent.

\subsection{Notations}

For $n \geqslant 1$, we work on $\mathbb{R}^{n}$ or $\mathbb{C}^{n}$ which are equipped with their canonical Euclidean structure. The spaces $\mathcal{M}_{n}(\mathbb{R})$ and $\mathcal{M}_{n}(\mathbb{C})$ of $n \times n$ matrices with real or complex entries are equipped with the associated Euclidean structure defined by $\|T\|_{\mathrm{HS}}^{2}=\operatorname{tr}\left(T^{\star} T\right)$ for any $T \in \mathcal{M}_{n}$, embedded in $\mathbb{R}^{n^{2}}$ or $\mathbb{R}^{2 n^{2}}$ endowed with the Lebesgue measure denoted by $\mathrm{d} T$. For every $x \in \mathbb{R}^{n}$, let

$$
\|x\|_{p}=\left(\sum_{i=1}^{n}\left|x_{i}\right|^{p}\right)^{1 / p} \quad \text { for } 1 \leqslant p<\infty \text { and }\|x\|_{\infty}=\max _{i \leqslant n}\left|x_{i}\right| .
$$

For any matrix $T \in \mathcal{M}_{n}(\mathbb{R})$ or $T \in \mathcal{M}_{n}(\mathbb{C})$, let $s(T)=\left(s_{1}(T), \ldots, s_{n}(T)\right)$ be the non-increasing rearrangement of the singular values of $T$ i.e. the eigenvalues of $\left(T^{*} T\right)^{1 / 2}$. For every $1 \leqslant p \leqslant \infty$, we define $\sigma_{p}(T)=\|s(T)\|_{p}$. Let $S_{p}^{n}$ be the Schatten trace class of matrices on the $n$-dimensional Euclidean space equipped with the norm $\sigma_{p}$, and denote

$$
B\left(S_{p}^{n}\right)=\left\{T \in \mathcal{M}_{n} ; \sigma_{p}(T) \leqslant 1\right\} .
$$

Of course, $\sigma_{2}(T)=\|T\|_{\mathrm{HS}}=\|T\|_{B\left(S_{2}^{n}\right)}$. We denote by $|A|$ the Lebesgue measure of any Borel set $A \subseteq S_{p}^{n}$, when it is finite and when $0<|A|<+\infty$, we denote $\tilde{A}=\lambda A$ where $\lambda$ is chosen such that $|\tilde{A}|=1$.

Whenever we write $a \simeq b$ we mean that there exist universal constants $c_{1}, c_{2}>0$ such that $c_{1} a \leqslant b \leqslant c_{2} a$.

\section{Reduction to integrals over $\mathbb{R}^{n}$}

A function $F: \mathbb{R}^{n} \rightarrow \mathbb{R}$ is said to be a symmetric function if for every permutation $\pi$ on $\{1, \ldots, n\}$,

$$
F\left(x_{1}, \ldots, x_{n}\right)=F\left(x_{\pi(1)}, \ldots, x_{\pi(n)}\right) .
$$

The function $F: \mathbb{R}^{n} \rightarrow \mathbb{R}$ is said to be positively homogeneous of degree $k \in \mathbb{R}$ if for every $\alpha \in \mathbb{R}_{+}^{*}$,

$$
F\left(\alpha x_{1}, \ldots, \alpha x_{n}\right)=\alpha^{k} F\left(x_{1}, \ldots, x_{n}\right) .
$$

Following the book of Mehta [12], it is natural to consider the spaces of real self-adjoint matrices, complex Hermitian matrices, antisymmetric Hermitian matrices, symplectic matrices and we refer to this book for the definitions of these ensembles. We will denote by $E$ the subspace of $\mathcal{M}_{n}$ that defines these ensemble (whatever is the field of the considered manifolds, real or complex). In each of these cases, it is well known [12] that there exists $a, b$ positive integer, a nonnegative integer $c$ and a constant $c_{n}$ depending only on $n, a, b, c$ such that for any symmetric continuous function $F: \mathbb{R}^{n} \rightarrow \mathbb{R}$

$$
\int_{T \in B\left(S_{p}^{n}\right) \cap E} F\left(s_{1}(T), \ldots, s_{n}(T)\right) \mathrm{d} T=c_{n} \int_{B_{p}^{n}} F(x) f_{a, b, c}(x) \mathrm{d} x
$$

where

$$
f_{a, b, c}(x)=\prod_{1 \leqslant j<i \leqslant n}\left|x_{i}^{a}-x_{j}^{a}\right|^{b} \times \prod_{i=1}^{n}\left|x_{i}\right|^{c} \quad \text { and } \quad B_{p}^{n}=\left\{x \in \mathbb{R}^{n},\|x\|_{p} \leqslant 1\right\} .
$$


A similar type of change of variables is discussed and explained in the work of Saint-Raymond [17]. He proved that the same result holds when we consider the space $E$ of complex (or real) matrices [17] with $a=2, b=2$, $c=1$ (or $a=2, b=1, c=0$ ). Since $f_{a, b, c}$ is positively homogeneous of degree $a b n(n-1) / 2+c n$, define $d_{n}$ by $d_{n}=\operatorname{abn}(n-1) / 2+(c+1) n$, then in each of these examples, $d_{n}=\operatorname{dim} E$. We will use the following lemma which is exactly in the same spirit as Lemma 1 in [11].

Lemma 1. For any symmetric continuous function $F: \mathbb{R}^{n} \rightarrow \mathbb{R}$, positively homogeneous of degree $k$, for every $p \geqslant 1$, one has

$$
\int_{T \in B\left(S_{p}^{n}\right) \cap E} F\left(s_{1}(T), \ldots, s_{n}(T)\right) \mathrm{d} T=\frac{c_{n}}{\Gamma\left(1+\left(d_{n}+k\right) / p\right)} \int_{\mathbb{R}^{n}} F(x) \mathrm{e}^{-\|x\|_{p}^{p}} f_{a, b, c}(x) \mathrm{d} x .
$$

Proof. We recall here a proof of this result for reason of completeness. It follows from Fubini's theorem:

$$
\begin{aligned}
\int_{\mathbb{R}^{n}} F(x) \mathrm{e}^{-\|x\|_{p}^{p}} f_{a, b, c}(x) \mathrm{d} x & =\int_{\mathbb{R}^{n}} F(x) f_{a, b, c}(x)\left(\int_{\|x\|_{p}^{p}}^{+\infty} \mathrm{e}^{-t} \mathrm{~d} t\right) \mathrm{d} x \\
& =\int_{0}^{+\infty} \mathrm{e}^{-t}\left(\int_{\left\{x,\|x\|_{p} \leqslant t^{1 / p}\right\}} F(x) f_{a, b, c}(x) \mathrm{d} x\right) \mathrm{d} t \\
& =\Gamma\left(1+\frac{d_{n}+k}{p}\right) \int_{B_{p}^{n}} F(x) f_{a, b, c}(x) \mathrm{d} x
\end{aligned}
$$

since $F$ is positively homogeneous of degree $k$ and $f_{a, b, c}$ of degree $d_{n}-n$. We conclude using (3).

Next, we need some estimates about the volume of the sets $B\left(S_{p}^{n}\right) \cap E$. Exact computations of these volumes are done in the paper of Saint-Raymond [17] for the unit balls of matrices of $S_{p}^{n}$ with real entries or complex entries. In the next proposition, we give a proof of an estimate of $\left(\left|B\left(S_{p}^{n}\right) \cap E\right|\right)^{1 / d_{n}}$ valid for every ensembles of matrices that we consider in this paper. Recall that in each case, the dimension of $E, d_{n}$, is equivalent to $n^{2}$ (up to constants depending only on $a$ and $b$ ). We will need the following classical estimate about the operator norm of a random Gaussian operator in $E$.

Lemma 2. Let $E$ be one of the classical ensemble considered in this paper. Let $G_{E}$ be a Gaussian vector in E. There exists a constant $c \geqslant 2$ such that for every integer $n$,

$$
\mathbb{E}\left\|G_{E}\right\|_{B\left(S_{\infty}^{n}\right)} \leqslant c \sqrt{n} .
$$

Proof. By Chevet's inequality [9], it is well known that $\mathbb{E}\left\|G_{\mathcal{M}_{n}(\mathbb{R})}\right\|_{B\left(S_{\infty}^{n}\right)} \leqslant 2 \sqrt{2 n}$ when $G_{\mathcal{M}_{n}(\mathbb{R})}$ is a $n \times n$ Gaussian matrix with independent real Gaussian $\mathcal{N}(0,1)$ entries. In the case of complex entries, we decompose the matrix as sum of its real and imaginary part and use triangle inequality to get that $\mathbb{E}\left\|G_{\mathcal{M}_{n}(\mathbb{C})}\right\|_{B\left(S_{\infty}^{n}\right)} \leqslant 4 \sqrt{2 n}$. In the case of quaternionic entries, we separate it in a sum of four terms and get that $\mathbb{E}\left\|G_{\mathcal{M}_{n}(\mathbb{H})}\right\|_{B\left(S_{\infty}^{n}\right)} \leqslant 8 \sqrt{2 n}$. We can also refer to the survey [10] where better constants are obtained. Now, let $\Sigma$ be one of the field $\mathbb{R}, \mathbb{C}$, or $\mathbb{H}$ then for every ensemble $E$, it is clear that if we denote by $G_{E}$ a Gaussian vector in $E$ then $G_{E}=P_{E}\left(G_{\mathcal{M}_{n}(\Sigma)}\right)$ where $P_{E}$ is the orthogonal projection on $E$. Since an orthogonal projection has norm 1, the estimate of the lemma follows.

We are now able to state the proposition.

Proposition 3. For every $p \geqslant 1$, up to constants depending only on $a$ and $b$,

$$
\left(\left|B\left(S_{p}^{n}\right) \cap E\right|\right)^{1 / d_{n}} \simeq d_{n}^{-1 / 4-1 / 2 p} \simeq n^{-1 / 2-1 / p} .
$$


Proof. Let $K$ be a symmetric convex body in $\mathbb{R}^{d}$ and $B_{2}^{d}$ the Euclidean ball in $\mathbb{R}^{d}$ then by Urysohn's inequality [16], we get that

$$
\left(\frac{|K|}{\left|B_{2}^{d}\right|}\right)^{1 / d} \leqslant \frac{c}{\sqrt{d}} \mathbb{E}\|G\|_{K^{o}}
$$

where $G$ is a Gaussian vector in $\mathbb{R}^{d}$ with independent $\mathcal{N}(0,1)$ entries, $K^{o}=\left\{y \in \mathbb{R}^{d}, \forall x \in K,\langle x, y\rangle \leqslant 1\right\}$ denotes the polar of $K$ and $c$ is a universal constant. Since $\left|B_{2}^{d}\right|^{1 / d} \simeq 1 / \sqrt{d}$, we get that for every symmetric convex body $K \subset \mathbb{R}^{d}$,

$$
|K|^{1 / d} \leqslant c_{2}^{\prime} \frac{\mathbb{E}\|G\|_{K^{o}}}{d} .
$$

Moreover, by reverse Santaló inequality [7] there exist universal constant $c_{1}$ such that

$$
\left(\frac{\left|B_{2}^{d}\right|}{|K|}\right)^{1 / d} \leqslant c_{1}\left(\frac{\left|K^{o}\right|}{\left|B_{2}^{d}\right|}\right)^{1 / d} .
$$

Using again Urysohn's inequality for $K^{o}$ and the fact that $\left|B_{2}^{d}\right|^{1 / d} \simeq 1 / \sqrt{d}$, there is a universal constant $c_{2}$ such that

$$
\frac{c_{2}}{\mathbb{E}\|G\|_{K}} \leqslant|K|^{1 / d} .
$$

Therefore, we get that for every symmetric convex body $K \subset \mathbb{R}^{d}$,

$$
\frac{c_{2}}{\mathbb{E}\|G\|_{K}} \leqslant|K|^{1 / d} \leqslant c_{2}^{\prime} \frac{\mathbb{E}\|G\|_{K^{o}}}{d} .
$$

We will apply this inequality in the situation we are interested in. Let $K=B\left(S_{p}^{n}\right) \cap E$ and $B_{2}^{d}=B_{2}^{d_{n}}=B\left(S_{2}^{n}\right) \cap E$. Remark that the norm in $S_{\infty}^{n}$ corresponds to the operator norm. By Hölder's inequality, for any matrix $T \in E$, for every $p \geqslant 1$,

$$
\|T\|_{B\left(S_{p}^{n}\right)} \leqslant n^{1 / p}\|T\|_{B\left(S_{\infty}^{n}\right)} \quad \text { and } \quad\|T\|_{\left(B\left(S_{p}^{n}\right) \cap E\right)^{o}} \leqslant\|T\|_{B\left(S_{p^{*}}^{n}\right)}
$$

where $1 / p+1 / p^{*}=1$. Let $G_{E}$ be a Gaussian vector in $E$ then, by Lemma 2, there exists a constant $c>0$ such that for every $n, \mathbb{E}\left\|G_{E}\right\|_{B\left(S_{\infty}^{n}\right)} \leqslant c \sqrt{n}$. Therefore,

$$
\mathbb{E}\left\|G_{E}\right\|_{K^{o}}=\mathbb{E}\left\|G_{E}\right\|_{\left(B\left(S_{p}^{n}\right) \cap E\right)^{o}} \leqslant \mathbb{E}\left\|G_{E}\right\|_{B\left(S_{p^{*}}^{n}\right)} \leqslant n^{1 / p^{*}} \mathbb{E}\left\|G_{E}\right\|_{B\left(S_{\infty}^{n}\right)} \leqslant c n^{3 / 2-1 / p}
$$

where $1 / p+1 / p^{*}=1$, and

$$
\mathbb{E}\left\|G_{E}\right\|_{K}=\mathbb{E}\left\|G_{E}\right\|_{B\left(S_{p}^{n}\right)} \leqslant n^{1 / p} \mathbb{E}\left\|G_{E}\right\|_{B\left(S_{\infty}^{n}\right)} \leqslant c n^{1 / 2+1 / p} .
$$

Since $d_{n} \simeq n^{2}$, this proves the estimate (4).

We are now able to explain how the estimates of the $L_{q}$ norms in Theorem 1 is related to the estimate of integrals with respect to the measure $M_{a, b, c, p}$ defined by

$$
M_{a, b, c, p}(f)=\int_{\mathbb{R}^{n}} f(x) f_{a, b, c}(x) \mathrm{e}^{-\|x\|_{p}^{p}} \mathrm{~d} x .
$$

Lemma 4. Let $E$ be any of the classical space of matrices on the real or complex field (the space of real, complex matrices, real self-adjoint matrices, complex Hermitian matrices, antisymmetric Hermitian matrices or symplectic matrices), and let $a, b$ and $c$ the corresponding integers associate to the ensemble defined by (3) and let $d_{n}=\operatorname{dim} E=$ abn $(n-1) / 2+(c+1) n$. Then for every $1 \leqslant p<\infty$, if we define $M_{a, b, c, p}$ as the measure of density $f_{a, b, c}(x) \mathrm{e}^{-\|x\|_{p}^{p}}$, for every $1 \leqslant q \leqslant d_{n}$,

$$
I_{q}\left(B\left(S_{p}^{n}\right) \cap E, B\left(S_{2}^{n}\right) \cap E\right) \simeq n^{1 / 2-1 / p}\left(\frac{M_{a, b, c, p}\left(\|x\|_{2}^{q}\right)}{M_{a, b, c, p}(1)}\right)^{1 / q}
$$

where the constants in this equivalence depend only on $a, b, c$. 
Proof. For notational convenience, we will write $I_{q}\left(B\left(S_{p}^{n}\right) \cap E\right)$ instead of

$$
I_{q}\left(B\left(S_{p}^{n}\right) \cap E, B\left(S_{2}^{n}\right) \cap E\right) .
$$

Using Lemma 1 for the functions $F=1$ and $F(x)=\|x\|_{2}^{q}$, we get

$$
\left|B\left(S_{p}^{n}\right) \cap E\right|=\int_{T \in B\left(S_{p}^{n}\right) \cap E} \mathrm{~d} T=\frac{c_{n}}{\Gamma\left(1+d_{n} / p\right)} \int_{\mathbb{R}^{n}} f_{a, b, c}(x) \mathrm{e}^{-\|x\|_{p}^{p}} \mathrm{~d} x,
$$

and

$$
\begin{aligned}
I_{q}\left(B\left(S_{p}^{n}\right) \cap E\right) & =\left|B\left(S_{p}^{n}\right) \cap E\right|^{-1 / d_{n}}\left(\left|B\left(S_{p}^{n}\right) \cap E\right|^{-1} \int_{T \in B\left(S_{p}^{n}\right) \cap E}\left(\sum_{i=1}^{n}\left|\sigma_{i}(T)\right|^{2}\right)^{q / 2} \mathrm{~d} T\right)^{1 / q} \\
& =\left|B\left(S_{p}^{n}\right) \cap E\right|^{-1 / d_{n}}\left(\left|B\left(S_{p}^{n}\right) \cap E\right|^{-1} \frac{c_{n}}{\Gamma\left(1+\left(d_{n}+q\right) / p\right)} \int_{\mathbb{R}^{n}}\|x\|_{2}^{q} f_{a, b, c}(x) \mathrm{e}^{-\|x\|_{p}^{p}} \mathrm{~d} x\right)^{1 / q} \\
& =\left|B\left(S_{p}^{n}\right) \cap E\right|^{-1 / d_{n}}\left(\frac{c_{n} \Gamma\left(1+d_{n} / p\right)}{c_{n} \Gamma\left(1+\left(d_{n}+q\right) / p\right)}\right)^{1 / q}\left(\frac{\int_{\mathbb{R}^{n}}\|x\|_{2}^{q} f_{a, b, c}(x) \mathrm{e}^{-\|x\|_{p}^{p}} \mathrm{~d} x}{\int_{\mathbb{R}^{n}} f_{a, b, c}(x) \mathrm{e}^{-\|x\|_{p}^{p}} \mathrm{~d} x}\right)^{1 / q} .
\end{aligned}
$$

We have proved in (4) that $\left|B\left(S_{p}^{n}\right) \cap E\right|^{1 / d_{n}} \simeq n^{-1 / 2-1 / p}$ and since

$$
\left(\frac{\Gamma\left(1+d_{n} / p\right)}{\Gamma\left(1+\left(d_{n}+q\right) / p\right)}\right)^{1 / q} \simeq d_{n}^{-1 / p}
$$

we get the claimed estimate (because $d_{n} \simeq n^{2}$ ).

For the measures $M_{a, b, c, p}$, we can provide exact computations of some integrals which are presented in the next section. The result which is of interest for our problem is as follows and will be proved in the next section.

Lemma 5. For every integers $a, b, c$, there are positive constants $c_{1}$ and $c_{2}$ such that for every $n \in \mathbb{N}$, for every $1 \leqslant p \leqslant 2$, define the measures $M_{a, b, c, p}$ as above, then, for every $2 \leqslant q \leqslant c_{1} n^{1+p / 2}$,

$$
\left(\frac{M_{a, b, c, p}\left(\|x\|_{2}^{q}\right)}{M_{a, b, c, p}(1)}\right)^{1 / q} \leqslant c_{2} n^{1 / 2+1 / p} .
$$

Proof of Theorem 1. It is well known (see [13]) that for every symmetric convex body $K \subset \mathbb{R}^{d}$,

$$
\left(\frac{1}{|K|^{1+2 / d}} \int_{K}\|x\|_{2}^{2} \mathrm{~d} \lambda(x)\right)^{1 / 2} \geqslant\left(\frac{1}{\left|B_{2}^{d}\right|^{1+2 / d}} \int_{B_{2}^{d}}\|x\|_{2}^{2} \mathrm{~d} \lambda(x)\right)^{1 / 2} \geqslant C \sqrt{d}
$$

where $B_{2}^{d}$ is the Euclidean ball associated to $\|\cdot\|_{2}$ and $C$ is a universal constant.

When $p \geqslant 2$, the assertion of the theorem follows from the following observation:

For every symmetric convex body $K \subset \mathbb{R}^{d}, I_{d}\left(K, B_{2}^{d}\right) \simeq \max _{x \in \widetilde{K}}\|x\|_{2}$.

Indeed,

$$
I_{d}\left(K, B_{2}^{d}\right)=\left(\int_{\widetilde{K}}\|x\|_{2}^{d} \mathrm{~d} x\right)^{1 / d} \simeq\left(\int_{S^{d-1}} \int_{\widetilde{K}}|\langle x, \theta\rangle|^{d} \mathrm{~d} x \mathrm{~d} \sigma(\theta)\right)^{1 / d} .
$$

Since $\theta \mapsto\left(\int_{\widetilde{K}}|\langle x, \theta\rangle|^{d} \mathrm{~d} x\right)^{1 / d}$ defines a norm on $\mathbb{R}^{d}$, it is well known using concentration of measure on the sphere [14] that

$$
I_{d}(K, \mathcal{E}) \simeq\left(\max _{\theta \in S^{d-1}} \int_{\widetilde{K}}|\langle x, \theta\rangle|^{d} \mathrm{~d} x\right)^{1 / d}
$$


and a classical use of Brunn-Minkowski inequality (see [6]) proves that for every symmetric convex body $\widetilde{K}$,

$$
\left(\int_{\widetilde{K}}|\langle x, \theta\rangle|^{d} \mathrm{~d} x\right)^{1 / d} \geqslant c \max _{x \in \widetilde{K}}|\langle x, \theta\rangle|
$$

which finishes the proof of (5).

Let $E$ be one of the classical ensemble of matrices, $d_{n}=\operatorname{dim} E$ where $d_{n}=a b n(n-1) / 2+(c+1) n$ and $a, b, c$ are defined in (3). Let $K=B\left(S_{p}^{n}\right) \cap E$ and $\mathcal{E}=B\left(S_{2}^{n}\right) \cap E=B_{2}^{d_{n}}$.

If $p \geqslant 2$ then by (5), $I_{d_{n}}(K, \mathcal{E}) \simeq \max _{T \in \widetilde{K}}\|T\|_{\mathrm{HS}}$ and by (4), we conclude that there are constants $C, C^{\prime}>0$ (depending only on $a, b, c)$ such that $C n \leqslant I_{2}(K, \mathcal{E}) \leqslant I_{d_{n}}(K, \mathcal{E}) \leqslant C^{\prime} n$ which proves the assertion of the theorem.

If $1 \leqslant p \leqslant 2$, then by Lemma 4

$$
\left(\frac{1}{|K|^{1+q / d_{n}}} \int_{K}\|T\|_{\mathrm{HS}}^{q} \mathrm{~d} \lambda(T)\right)^{1 / q} \simeq n^{1 / 2-1 / p}\left(\frac{M_{a, b, c, p}\left(\|x\|_{2}^{q}\right)}{M_{a, b, c, p}(1)}\right)^{1 / q} .
$$

By Lemma 5, we get that there exist $c_{1}$ and $c_{2}$ depending only on $a, b, c$ such that for every $n$, for every $2 \leqslant q \leqslant$ $c_{1} n^{1+p / 2}$,

$$
C \sqrt{d_{n}} \leqslant\left(\frac{1}{|K|^{1+q / d_{n}}} \int_{K}\|T\|_{\mathrm{HS}}^{q} \mathrm{~d} \lambda(T)\right)^{1 / q} \leqslant c_{2} n .
$$

This ends the proof of the theorem.

\section{Computation of integrals with respect to the measures $M_{a, b, c, p}$}

First we need some precise results concerning the computation of the integrals of positively homogeneous functions with respect to the measures $M_{a, b, c, p}$. We shall prove a generalization of Lemma 3 in [11], which is based on a method developed by Aomoto [3] for the study of Jacobi polynomials associated to Selberg integrals. We start by recalling the notations. Let $a, b \in \mathbb{N}^{*}, c \in \mathbb{N}$ and $f_{a, b, c}: \mathbb{R}^{n} \rightarrow \mathbb{R}$ defined by

$$
f_{a, b, c}(x)=f_{a, b, c}\left(x_{1}, \ldots, x_{n}\right)=\prod_{1 \leqslant j<i \leqslant n}\left|x_{i}{ }^{a}-x_{j}{ }^{a}\right|^{b} \times \prod_{i=1}^{n}\left|x_{i}\right|^{c} .
$$

Denote by $M_{p, a, b, c}=M_{p}$ the measure in $\mathbb{R}^{n}$ with density

$$
f_{a, b, c, p}\left(x_{1}, \ldots, x_{n}\right)=f_{a, b, c}(x) \exp \left(-\sum_{i=1}^{n}|x|_{i}^{p}\right)
$$

i.e. for every $f: \mathbb{R}^{n} \rightarrow \mathbb{R}$,

$$
M_{p}(f)=\int_{\mathbb{R}^{n}} f(x) f_{a, b, c}(x) \mathrm{e}^{-\sum_{i=1}^{n}|x|_{i}^{p}} \mathrm{~d} x .
$$

Denote by $d_{n}=a b n(n-1) / 2+(c+1) n$. Observe that $f_{a, b, c}$ is a positively homogeneous function of order $a b n(n-1) / 2+c n$. Note also that for every function $g: \mathbb{R}^{n} \rightarrow \mathbb{R}$ positively homogeneous of order $s>-n$ such that $\int_{S^{n-1}}|g(u)| \mathrm{d} \sigma(u)<+\infty$, for every $p \geqslant 1, \int_{\mathbb{R}^{n}}|g(x)| \exp \left(-\|x\|_{p}^{p}\right) \mathrm{d} x$ is finite.

Lemma 6. Let $\beta \geqslant 0, s \geqslant-d_{n}-\beta$ and $f: \mathbb{R}^{n} \rightarrow \mathbb{R}_{+}$a positively homogeneous function of order $s$ and $C^{1}$ on $\mathbb{R}^{n} \backslash\{0\}$. Then

$$
\begin{aligned}
(\beta+c+1) M_{p}\left(\left(\sum_{i=1}^{n}\left|x_{i}\right|^{\beta}\right) f(x)\right)= & p M_{p}\left(\|x\|_{\beta+p}^{\beta+p} f(x)\right)-M_{p}\left(\sum_{i=1}^{n}\left|x_{i}\right|^{\beta} x_{i} \frac{\partial f}{\partial x_{i}}(x)\right) \\
& -a b M_{p}\left(\left(\sum_{i=1}^{n} \sum_{k \neq i} \frac{\left|x_{i}\right|^{\beta} x_{i}^{a}}{x_{i}^{a}-x_{k}^{a}}\right) f(x)\right) .
\end{aligned}
$$


Proof. For fixed $x_{2}, \ldots, x_{n}$ we define $g_{1}: \mathbb{R} \rightarrow \mathbb{R}_{+}$by

$$
g_{1}\left(x_{1}\right)=\left|x_{1}\right|^{\beta} x_{1} f\left(x_{1}, x_{2}, \ldots, x_{n}\right) f_{a, b, c}\left(x_{1}, x_{2}, \ldots, x_{n}\right) \exp \left(-\left|x_{1}\right|^{p}\right) \exp \left(-\sum_{j=2}^{n}\left|x_{j}\right|^{p}\right) .
$$

Since $f$ is positively homogeneous, $\lim _{x_{1} \rightarrow+\infty} g_{1}\left(x_{1}\right)=0$ and $\lim _{x_{1} \rightarrow-\infty} g_{1}\left(x_{1}\right)=0$. Without loss of generality, we can assume that $x_{2}<x_{3}<\cdots<x_{n}$ therefore $g_{1}^{\prime}$ is continuous on $\mathbb{R} \backslash\left\{0, x_{2}, \ldots, x_{n}\right\}$ and has finite left and right limits at each of these points of discontinuity. Observe also that $g_{1}(x)=0$ for every $x \in\left\{0, x_{2}, \ldots, x_{n}\right\}$. Integration by parts proves the following equality, for almost all $\left(x_{2}, \ldots, x_{n}\right)$ with respect to the Lebesgue measure in $\mathbb{R}^{n-1}$ :

$$
\begin{aligned}
& \int_{-\infty}^{\infty}\left|x_{1}\right|^{\beta} f(x) \exp \left(-\sum_{j=1}^{n}\left|x_{j}\right|^{p}\right) f_{a, b, c}(x) \mathrm{d} x_{1} \\
& =-\frac{1}{\beta+1} \int_{-\infty}^{\infty}\left|x_{1}\right|^{\beta} x_{1} \frac{\partial}{\partial x_{1}}\left(f(x) \exp \left(-\sum_{j=1}^{n}\left|x_{j}\right|^{p}\right) f_{a, b, c}(x)\right) \mathrm{d} x_{1} .
\end{aligned}
$$

For every $x_{1} \notin\left\{x_{2}, \ldots, x_{n}\right\}$, we can assume that there exists $2 \leqslant m \leqslant n-1$ such that $x_{1} \in\left(x_{m}, x_{m+1}\right)$ (the cases $x_{1}<x_{2}$ and $x_{1}>x_{n}$ are handled similarly). Then,

$$
\prod_{1 \leqslant j<i \leqslant n}\left|x_{i}^{a}-x_{j}^{a}\right|^{b}=\prod_{2 \leqslant j \leqslant m}\left(x_{j}^{a}-x_{1}^{a}\right)^{b} \prod_{m+1 \leqslant j \leqslant n}\left(x_{1}^{a}-x_{j}^{a}\right)^{b} \prod_{2 \leqslant j<i \leqslant n}\left|x_{i}^{a}-x_{j}^{a}\right|^{b}
$$

and it is easy to check that

$$
\frac{\partial f_{a, b, c}(x)}{\partial x_{1}}=\left(a b\left(\sum_{k \geqslant 1} \frac{x_{1}^{a-1}}{x_{1}^{a}-x_{k}^{a}}\right)+\frac{c}{x_{1}}\right) f_{a, b, c}(x) .
$$

Combining with the integral identity above, we get that for almost all $\left(x_{2}, \ldots, x_{n}\right)$

$$
\begin{aligned}
(\beta+ & 1) \int_{-\infty}^{\infty}\left|x_{1}\right|^{\beta} f(x) \exp \left(-\|x\|_{p}^{p}\right) f_{a, b, c}(x) \mathrm{d} x_{1} \\
= & p \int_{-\infty}^{\infty}\left|x_{1}\right|^{\beta+p} f(x) \exp \left(-\|x\|_{p}^{p}\right) f_{a, b, c}(x) \mathrm{d} x_{1}-\int_{-\infty}^{\infty}\left|x_{1}\right|^{\beta} x_{1}\left(\frac{\partial f}{\partial x_{1}}(x)\right) \exp \left(-\|x\|_{p}^{p}\right) f_{a, b, c}(x) \mathrm{d} x_{1} \\
& -\int_{-\infty}^{\infty}\left(\left(a b \sum_{k \geqslant 1} \frac{\left|x_{1}\right|^{\beta} x_{1}^{a}}{x_{1}^{a}-x_{k}^{a}}\right)+c\left|x_{1}\right|^{\beta}\right) f(x) \exp \left(-\|x\|_{p}^{p}\right) f_{a, b, c}(x) \mathrm{d} x_{1} .
\end{aligned}
$$

Integration with respect to $x_{2}, \ldots, x_{n}$ and the definition of $M_{p}$ give

$$
(\beta+c+1) M_{p}\left(\left|x_{1}\right|^{\beta} f(x)\right)=p M_{p}\left(\left|x_{1}\right|^{\beta+p} f(x)\right)-M_{p}\left(\left|x_{1}\right|^{\beta} x_{1} \frac{\partial f}{\partial x_{i}}(x)\right)-a b M_{p}\left(\left(\sum_{k \geqslant 1} \frac{\left|x_{1}\right|^{\beta} x_{1}^{a}}{x_{1}^{a}-x_{k}^{a}}\right) f(x)\right) .
$$

This is obviously valid for every $i=1, \ldots, n$ and summing these equalities we get

$$
\begin{aligned}
(\beta+c+1) M_{p}\left(\left(\sum_{i=1}^{n}\left|x_{1}\right|^{\beta}\right) f(x)\right)= & p M_{p}\left(\|x\|_{\beta+p}^{\beta+p} f(x)\right)-M_{p}\left(\sum_{i=1}^{n}\left|x_{i}\right|^{\beta} x_{i} \frac{\partial f}{\partial x_{i}}(x)\right) \\
& -a b M_{p}\left(\left(\sum_{i=1}^{n} \sum_{k \neq i} \frac{\left|x_{i}\right|^{\beta} x_{i}^{a}}{x_{i}^{a}-x_{k}^{a}}\right) f(x)\right) .
\end{aligned}
$$

Corollary 7. With the notations defined at the beginning of this section, we have: 
(a) if $f$ is a positively homogeneous function of degree $s$, integrable on $S^{n-1}$ and $s>-d_{n}$ then

$$
\left(d_{n}+s\right) M_{p}(f(x))=p M_{p}\left(\|x\|_{p}^{p} f(x)\right) ;
$$

(b) for any $q>-d_{n}-2$,

$$
p M_{p}\left(\|x\|_{p+2}^{p+2}\|x\|_{2}^{q}\right)-q M_{p}\left(\|x\|_{4}^{4}\|x\|_{2}^{q-2}\right) \leqslant \zeta_{(a, 2)}\left(\frac{d_{n}}{n}+2\right) M_{p}\left(\|x\|_{2}^{q+2}\right)
$$

where $\zeta_{(1,2)}=\frac{3}{2}$ and $\zeta_{(a, 2)}=1$ for every $a \geqslant 2$

(c) for any $q>-d_{n}-p$,

$$
p M_{p}\left(\|x\|_{2 p}^{2 p}\|x\|_{2}^{q}\right)-q M_{p}\left(\|x\|_{p+2}^{p+2}\|x\|_{2}^{q-2}\right) \leqslant \zeta_{(a, p)}\left(\frac{d_{n}}{n}+p\right) M_{p}\left(\|x\|_{p}^{p}\|x\|_{2}^{q}\right)
$$

where $\zeta_{(a, p)}=\max \left\{\frac{a+p}{2 a}, 1\right\}$.

Remark. Simple observations in the proof below show that the two inequalities established in (b) and (c) can be reversed with other constants (independent of the dimension $n$ ).

Proof. (a) Let $g: \mathbb{R} \rightarrow \mathbb{R}$ defined by

$$
g(t)=\int_{\mathbb{R}^{n}} f(x) f_{a, b, c}(x) \exp \left(-t\|x\|_{p}^{p}\right) \mathrm{d} x
$$

then $g$ is clearly a $C^{1}$ function on $\mathbb{R}$ and

$$
g^{\prime}(t)=-\int_{\mathbb{R}^{n}}\|x\|_{p}^{p} f(x) f_{a, b, c}(x) \exp \left(-t\|x\|_{p}^{p}\right) \mathrm{d} x .
$$

However, since $f$ is positively homogeneous of degree $s$ and $f_{a, b, c}$ is positively homogeneous of degree $a b n(n-$ $1) / 2+c n$ therefore, by the change of variable $y=t^{1 / p} x$ we get that

$$
g(t)=t^{-\left(s+d_{n}\right) / p} g(1) .
$$

Taking the derivative at 1 proves (a).

For (b) and (c) we will use Lemma 6, for $f(x)=\|x\|_{2}^{q}$. So observe that $\frac{\partial f}{\partial x_{i}}=q x_{i}\|x\|_{2}^{q-2}$.

One can check that for any positive integer $a$ and $\beta>0$, if $\zeta=\zeta_{(a, \beta)}=\max \left\{\frac{a+\beta}{2 a}, 1\right\}$ then,

$$
\forall x_{1} \neq x_{2}, \quad \frac{\left|x_{1}\right|^{\beta} x_{1}^{a}-\left|x_{2}\right|^{\beta} x_{2}^{a}}{x_{1}^{a}-x_{2}^{a}} \leqslant \zeta\left(\left|x_{1}\right|^{\beta}+\left|x_{2}\right|^{\beta}\right) .
$$

So, we get

$$
\sum_{i=1}^{n} \sum_{k \neq i} \frac{\left|x_{i}\right|^{\beta} x_{i}^{a}}{x_{i}^{a}-x_{k}^{a}}=\frac{1}{2}\left(\sum_{i=1}^{n} \sum_{k \neq i} \frac{\left|x_{1}\right|^{\beta} x_{1}^{a}-\left|x_{2}\right|^{\beta} x_{2}^{a}}{x_{1}^{a}-x_{2}^{a}}\right) \leqslant \frac{n-1}{2} \zeta\|x\|_{\beta}^{\beta} .
$$

To prove assertion (b) we apply Lemma 6 with $\beta=2$. So we get

$$
(c+3) M_{p}\left(\|x\|_{2}^{q+2}\right) \geqslant p M_{p}\left(\|x\|_{p+2}^{p+2}\|x\|_{2}^{q}\right)-q M_{p}\left(\|x\|_{4}^{4}\|x\|_{2}^{q-2}\right)-a b \zeta_{(a, 2)} \frac{n-1}{2} M_{p}\left(\|x\|_{2}^{q+2}\right) .
$$

To prove assertion (c) we apply Lemma 6 with $\beta=p$. So we get

$$
\begin{aligned}
(c+p+1) M_{p}\left(\|x\|_{p}^{p}\|x\|_{2}^{q}\right) \geqslant & p M_{p}\left(\|x\|_{2 p}^{2 p}\|x\|_{2}^{q}\right)-q M_{p}\left(\|x\|_{p+2}^{p+2}\|x\|_{2}^{q-2}\right) \\
& -a b \zeta_{(a, p)} \frac{n-1}{2} M_{p}\left(\|x\|_{p}^{p}\|x\|_{2}^{q}\right) .
\end{aligned}
$$

We conclude this section by proving the key estimate of Lemma 5. 
Proof of Lemma 5. Assume that $0 \leqslant q \leqslant d_{n}$. By Corollary 7(c) we have

$$
q M_{p}\left(\|x\|_{p+2}^{p+2}\|x\|_{2}^{q-2}\right) \geqslant p M_{p}\left(\|x\|_{2 p}^{2 p}\|x\|_{2}^{q}\right)-\zeta_{(a, p)}\left(\frac{d_{n}}{n}+p\right) M_{p}\left(\|x\|_{p}^{p}\|x\|_{2}^{q}\right) .
$$

On the other hand, by Corollary 7(b) (applied for $q-2$ ), we get

$$
\zeta_{(a, 2)}\left(\frac{d_{n}}{n}+2\right) M_{p}\left(\|x\|_{2}^{q}\right) \geqslant p M_{p}\left(\|x\|_{p+2}^{p+2}\|x\|_{2}^{q-2}\right)-(q-2) M_{p}\left(\|x\|_{4}^{4}\|x\|_{2}^{q-4}\right) .
$$

Combining the above, we get

$$
\begin{aligned}
& q \zeta_{(a, 2)}\left(\frac{d_{n}}{n}+2\right) M_{p}\left(\|x\|_{2}^{q}\right)+q(q-2) M_{p}\left(\|x\|_{4}^{4}\|x\|_{2}^{q-4}\right) \\
& \geqslant p^{2} M_{p}\left(\|x\|_{2 p}^{2 p}\|x\|_{2}^{q}\right)-p \zeta_{(a, p)}\left(\frac{d_{n}}{n}+p\right) M_{p}\left(\|x\|_{p}^{p}\|x\|_{2}^{q}\right) .
\end{aligned}
$$

Now, Corollary 7(a) applied to the function $f(x)=\|x\|_{2}^{q}$ gives

$$
\left(d_{n}+q\right) M_{p}\left(\|x\|_{2}^{q}\right)=p M_{p}\left(\|x\|_{p}^{p}\|x\|_{2}^{q}\right)
$$

which proves that

$$
\begin{aligned}
p^{2} M_{p}\left(\|x\|_{2 p}^{2 p}\|x\|_{2}^{q}\right) \leqslant & q(q-2) M_{p}\left(\|x\|_{4}^{4}\|x\|_{2}^{q-4}\right)+\left(\left(\frac{d_{n}}{n}+2\right) q \zeta_{(a, 2)}\right. \\
& \left.+\zeta_{(a, p)}\left(d_{n}+q\right)\left(\frac{d_{n}}{n}+p\right)\right) M_{p}\left(\|x\|_{2}^{q}\right) .
\end{aligned}
$$

Therefore, the following inequality holds for every $0 \leqslant q \leqslant d_{n}$ and every $p \geqslant 1$

$$
M_{p}\left(\|x\|_{2 p}^{2 p}\|x\|_{2}^{q}\right) \leqslant 6 \frac{d_{n}^{2}}{n} M_{p}\left(\|x\|_{2}^{q}\right)+q^{2} M_{p}\left(\|x\|_{4}^{4}\|x\|_{2}^{q-4}\right) .
$$

Using Corollary 7(a) with $f(x)=\|x\|_{4}^{4}\|x\|_{2}^{q-4}$, we get

$$
\left(d_{n}+q\right) M_{p}\left(\|x\|_{4}^{4}\|x\|_{2}^{q-4}\right)=p M_{p}\left(\|x\|_{p}^{p}\|x\|_{4}^{4}\|x\|_{2}^{q-4}\right) .
$$

By Hölder inequality, for every $x \in \mathbb{R}^{n}$ and $p \in[1,2]$,

$$
\|x\|_{p+2}^{p+2}\|x\|_{2}^{2} \geqslant n^{-1+p / 2}\|x\|_{4}^{4}\|x\|_{p}^{p}
$$

so

$$
M_{p}\left(\|x\|_{p+2}^{p+2}\|x\|_{2}^{q-2}\right) \geqslant n^{-1+p / 2} M_{p}\left(\|x\|_{4}^{4}\|x\|_{p}^{p}\|x\|_{2}^{q-4}\right)
$$

which proves by (7) that

$$
p M_{p}\left(\|x\|_{p+2}^{p+2}\|x\|_{2}^{q-2}\right) \geqslant n^{-1+p / 2}\left(d_{n}+q\right) M_{p}\left(\|x\|_{4}^{4}\|x\|_{2}^{q-4}\right) .
$$

Using again Corollary 7(b) (applied for $q-2$ ), we get

$$
\zeta_{(a, 2)}\left(\frac{d_{n}}{n}+2\right) M_{p}\left(\|x\|_{2}^{q}\right) \geqslant\left(\left(d_{n}+q\right) n^{-1+p / 2}-q+2\right) M_{p}\left(\|x\|_{4}^{4}\|x\|_{2}^{q-4}\right) .
$$

Therefore, we have proved that for every $0 \leqslant q \leqslant \frac{1}{2} n^{-1+p / 2} d_{n}$,

$$
M_{p}\left(\|x\|_{4}^{4}\|x\|_{2}^{q-4}\right) \leqslant 6 n^{-p / 2} M_{p}\left(\|x\|_{2}^{q}\right) .
$$

Combining (6) with (8), we conclude that for every $q \leqslant \frac{d_{n}}{2} n^{-1+p / 2}$

$$
M_{p}\left(\|x\|_{2}^{q}\|x\|_{2 p}^{2 p}\right) \leqslant 12 \frac{d_{n}^{2}}{n} M_{p}\left(\|x\|_{2}^{q}\right) .
$$


By symmetry of $M_{p}$ we have $M_{p}\left(x_{i}^{2 p}\|x\|_{2}^{q}\right) / M_{p}\left(\|x\|_{2}^{q}\right) \leqslant 12 d_{n}^{2} / n^{2}$, and Hölder's inequality shows that

$$
\frac{M_{p}\left(x_{i}^{2}\|x\|_{2}^{q}\right)}{M_{p}\left(\|x\|_{2}^{q}\right)} \leqslant\left(\frac{M_{p}\left(x_{i}^{2 p}\|x\|_{2}^{q}\right)}{M_{p}\left(\|x\|_{2}^{q}\right)}\right)^{1 / p} \leqslant 12\left(\frac{d_{n}}{n}\right)^{2 / p},
$$

which proves that for every $q \leqslant \frac{d_{n}}{2} n^{-1+p / 2}, M_{p}\left(\|x\|_{2}^{q+2}\right) / M_{p}\left(\|x\|_{2}^{q}\right) \leqslant 12 n\left(d_{n} / n\right)^{2 / p}$. Therefore

$$
\left(\frac{M_{p}\left(\|x\|_{2}^{q}\right)}{M_{p}(1)}\right)^{1 / q} \leqslant 2 \sqrt{3} n^{1 / 2}\left(\frac{d_{n}}{n}\right)^{1 / p}
$$

and this concludes the proof of lemma.

Remark. For $p=1$, the estimate of Theorem 1 is sharp up to a universal constant. Let $E$ be one of the classical ensemble of matrices, $d_{n}=\operatorname{dim} E$ where $d_{n}=a b n(n-1) / 2+(c+1) n$ and $a, b, c$ are defined in (3). Let $K=$ $B\left(S_{1}^{n}\right) \cap E$ and $\mathcal{E}=B\left(S_{2}^{n}\right) \cap E$. Let $I_{q}(K)=I_{q}(K, \mathcal{E})$.

Let $q_{0}$ be such that $I_{q_{0}}(K) \simeq n$. By a result of Carbery and Wright [8] we know that, for every $p, q \geqslant 1$ and every convex body $L, I_{p q}(L) \leqslant C p I_{q}(L)$, where $C>0$ is an absolute constant. Recall that by (5), we get in this case (using (4))

$$
I_{d_{n}}(K) \simeq \max _{x \in \widetilde{K}}\|x\|_{2} \simeq n^{3 / 2}
$$

therefore, necessarily

$$
n^{3 / 2} \leqslant c \frac{n^{2}}{q_{0}} I_{q_{0}}(K) \simeq \frac{n^{3}}{q_{0}} \quad \text { and } \quad q_{0} \leqslant c n^{3 / 2} .
$$

\section{Proof of Theorem 2}

In fact, Theorem 1 and Theorem 2 are equivalent. This is a consequence of the following more general but simple lemma (for a proof see [15]):

Lemma 8. There exists $C>1$ such that for $\phi \geqslant C$, for every convex body $K$ in $\mathbb{R}^{d}$ and for every $\gamma \geqslant 1$, the following are equivalent:

(a) For every $2 \leqslant q \leqslant \phi$,

$$
I_{q}(K, \mathcal{E}) \leqslant \gamma I_{2}(K, \mathcal{E})
$$

(b) For every $t \geqslant 1$,

$$
\lambda\left(\left\{x \in \widetilde{K}:\|x\|_{\mathcal{E}} \geqslant c_{2}(\gamma) I_{2}(K, \mathcal{E}) t\right\}\right) \leqslant \mathrm{e}^{-t c_{1} \phi}
$$

where $c_{2}(\gamma) \simeq \gamma$ and $c_{1}>0$ is a universal constant.

Also, in [15] an equivalent formulation of Lemma 8 can be found, showing that the initial conjecture (C) is related to the central limit properties of the convex bodies. We refer to [2,5] and [15] for more information in this direction.

\section{Acknowledgement}

We would like to thank G. Aubrun for several useful discussions and S. Szarek for the suggestion to generalize our earlier results to the case of classical ensembles of matrices.

\section{References}

[1] S. Alesker, $\psi_{2}$-estimate for the Euclidean norm on a convex body in isotropic position, in: Geometric Aspects of Functional Analysis (Israel, 1992-1994), Birkhäuser, Basel, 1995, pp. 1-4.

[2] M. Anttila, K. Ball, I. Perissinaki, The central limit problem for convex bodies, Trans. Amer. Math. Soc. 355 (12) (2003) $4723-4735$. 
[3] K. Aomoto, Jacobi polynomials associated with Selberg integrals, SIAM J. Math. Anal. 18 (2) (1987) 545-549.

[4] G. Aubrun, S. Szarek, Tensor products of convex sets and the volume of separable states on $N$ qudits, Preprint.

[5] S.G. Bobkov, F.L. Nazarov, On convex bodies and log-concave probability measures with unconditional basis, in: V.D. Milman, G. Schechtman (Eds.), Geom. Aspects of Funct. Analysis, in: Lecture Notes in Math., vol. 1807, Springer, 2003, pp. 44-52.

[6] C. Borell, Complements of Lyapunov's inequality, Math. Ann. 205 (1973) 323-331.

[7] J. Bourgain, V.D. Milman, New volume ratio properties for convex symmetric bodies in $R^{n}$, Invent. Math. 88 (2) (1987) $319-340$.

[8] A. Carbery, J. Wright, Distributional and $L^{q}$ norm inequalities for polynomials over convex bodies in $\mathbb{R}^{n}$, Math. Res. Lett. 8 (2001) $233-248$.

[9] S. Chevet, Séries de variables aléatoires gaussiennes à valeurs dans $E \hat{\otimes}_{\varepsilon} F$. Application aux produits d'espaces de Wiener abstraits, in: Séminaire sur la Géométrie des Espaces de Banach (1977-1978) Exp. 19, École Polytech., Palaiseau, 1978, p. 15.

[10] K.R. Davidson, S.J. Szarek, Local operator theory, random matrices and Banach spaces, in: Handbook of the Geometry of Banach Spaces, vol. 2, North-Holland, Amsterdam, 2003, pp. 1819-1820.

[11] H. König, M. Meyer, A. Pajor, The isotropy constants of the Schatten classes are bounded, Math. Ann. 312 (1998) $773-783$.

[12] M.L. Mehta, Random Matrices, second ed., Academic Press, Boston, 1991.

[13] V.D. Milman, A. Pajor, Isotropic position and inertia ellipsoids and zonoids of the unit ball of a normed $n$-dimensional space, in: J. Lindenstrauss, V.D. Milman (Eds.), Geom. Aspects of Funct. Analysis, in: Lecture Notes in Math., vol. 1376, Springer, 1989, pp. 64-104.

[14] V.D. Milman, G. Schechtman, Asymptotic Theory of Finite-Dimensional Normed Spaces, Lecture Notes in Math., vol. 1200, Springer, Berlin, 1986.

[15] G. Paouris, Concentration of mass and central limit properties of isotropic convex bodies, Proc. Amer. Math. Soc. 133 (2005) $565-575$.

[16] G. Pisier, The Volume of Convex Bodies and Banach Space Geometry, Cambridge Tracks in Math., vol. 94, Cambridge Univ. Press, 1989.

[17] J. Saint-Raymond, Le volume des idéaux d'opérateurs classiques, Studia Math. 80 (1984) 63-75. 